\title{
Randomized Control Trial Study On The Effect Of Health Education In Promoting Adherence To Treatment Among The Urban And Rural Tuberculosis Patients In Kenya
}

Humphrey Kimani Mbuti

Technical University of Kenya

Francis Ochieng Oguya ( $\square$ foguya@tukenya.ac.ke)

Technical University of Kenya https://orcid.org/0000-0002-4371-6354

Elizabeth Mwaniki

Technical University of Kenya

Peterson Warutere

Kenyatta University

Research article

Keywords: Health Education, Adherence to Treatment, Urban and Rural Tuberculosis Patients, Rural Health Facility and Urban Health Facility.

Posted Date: November 18th, 2020

DOl: https://doi.org/10.21203/rs.3.rs-110440/v1

License: (9) This work is licensed under a Creative Commons Attribution 4.0 International License.

Read Full License 


\section{Abstract}

Background: Tuberculosis is a global health concern and the incident rate in Kenya remains high. Because of the long duration of standard treatment (six months), there is a risk of treatment default by patients. Low adherence to treatment may result in the emergence of resistant strains of the Mycobacterium Tuberculosis in turn increasing mortality and prolonging the treatment duration. The rising TB cases in Kenya have been associated with poor adherence and low cure rate arising from inappropriate health education. Directly Observed Therapy, Short-course (DOTS) Strategy, in combination with patient education have proved to be more effective in reducing TB incident than the DOTS Strategy alone. However, there is lack of Evidence Based Protocol to guide Medical Professionals through the implementation of health education for TB patients.

Objective: The main objective of this study was to determine the effect of health education in promoting adherence to treatment among the urban and rural tuberculosis patients in Kenya. The study used the PRECEDE-PROCEED model.

Design: The study adopted Randomized Controlled Trial Design with pre-and post-test assessment. The Multi-Stage Sampling Technique was applied to select the study respondents. Random sampling was adopted to select the hospitals, health centers and dispensaries. Simple random sampling method was also used to assign the patients into experimental and control groups.

Setting: The study was conducted in fourteen public health facilities in Nairobi and Murang'a Counties; 2 Hospitals, 7 health centers, 5 dispensaries.

Participants: A total of 450 patients were recruited from the selected health facilities by random sampling according to probability proportionate to TB patient's population. Only 373 met the eligibility criteria for the study.

Intervention: Health Education Program for 10-15 minutes on average, twice a month for the next 6+ months as the Patient went for the weekly drug ration.

Main measurement outcome: Level of adherence to TB treatment in patients.

Methods: The study was conducted between September 2019 and February 2020. Only patients aged 18 years and above who had been on DOTS for at least two weeks were selected. Health education was given to those in the experimental group while those in the control group did not receive the intervention. After the six months of treatment the two groups were compared. Standard Questionnaire was used to collect data. Multivariate Analysis of Variance, Odds Ratio and Chi-square tests were used to evaluate the association between health education and adherence to TB treatment.

Results: 450 patients were recruited (experimental group=225, control group=225). 77 patients did not meet the eligibility criteria leaving 373 patients (experimental group $=186$, control group $=187$ ). $83.3 \%$ of patients in the experimental group had high level adherence after intervention in the post- test phase 
compared to $60.4 \%$ of patients who had high level adherence in the control group in the post-test phase. Wilk's $\Lambda$ had an F value of 18.540, $p<0.001$, Odds Ratio was 3.274 and $\chi^{2}=24.189, p<0.001$, indicating that the health education intervention improved adherence to medication. Gender, levels of education, marital status and primary occupation were also found to be significantly associated with adherence to medication $(p<0.05)$.

Conclusions: Health education enhanced patients' adherence to TB treatment regime. A health education program should be adopted and rolled out to health facilities and health care settings that provide TB services in Kenya.

\section{Background}

Despite being a curable disease and the availability of effective anti-tuberculosis agents for over thirty years, tuberculosis (TB) remains a major global public health concern with increasing incident rates. [1]. TB treatment spans over a long duration of standard treatment (six months). This poses the risk of treatment default by patients [2]. Adherence to long time treatment, like for tuberculosis, is very challenging because of its long term and drug related side effects. Unfortunately, low treatment adherence may result in the emergence of resistant strains of mycobacterium tuberculosis, increasing mortality and prolonging the treatment duration [3]. As is known, treatment adherence is very important for effective treatment outcomes and prevention of the drug resistant TB Bacilli Strain [2]. In order to improve the TB treatment adherence level, various interventions have been designed and implemented across the globe. Many studies on health education and adherence to tuberculosis treatment indicate that an appropriate health education program helps the patient complete the treatment regime and achieve treatment success [4]. A systematic review suggests that the effects of the DOTS Strategy can be strengthened by combining the Strategy with other interventions, such as provision of health education and incentives [5 Although evidence suggests health education intervention based on social and behavioral science theories are more effective than those without theoretical model base, most of the available interventional studies related to TB treatment adherence lack a health behavioral theoretical model base [4]. Kenya is one of those countries, where information on the appropriate health education intervention, based on social and behavioral science theories, are lacking. This is the basis for this study.

Ana et al. (2014), in their study in Panoda, Brazil, found a higher rate of treatment completion and improvement in TB knowledge, in homeless latent TB patients who received nurse case managed education with default tracking over a six months treatment program, as compared to those who received only one lecture on TB knowledge at the time of diagnosis [6].

Yan-Yan Liu (2017) found a significantly higher rate of attendance at follow up and general treatment adherence among patients receiving oral and written health education by pharmacists, as well as routine nursing and medical care in China [7]. Habyetes (2016) indicated significantly higher adherence to treatment in the intervention group who had received culture specific health education and culturally relevant education material as compared to those who had received no intervention [8]. 
Pornsak (2016), in a study conducted in Thailand to investigate the effectiveness of a health education program to improve TB patients' compliance during treatment established that with intervention, there was $76 \%$ success compared to $62 \%$ in the control group [4]. In this study, health behavior, life style, environment and health status significantly improved $(p<0.01)$. Further, with a comprehensive health education intervention and follow-up every two weeks in a TB clinic or tracing to the TB patient's house and work place, patients completed the treatment regime and achieved treatment success [4].

Andrew et al. (2019) established that lack of knowledge regarding the TB infection process and its treatment; contribute to feelings of helplessness and anxiety, in a similar study in India. With health education intervention, there was self-reported high compliance at $78 \%$ against $50 \%$ for the control group [9].

Edward et al. (2014) in their study to assess the effectiveness of interventions aimed at reducing TB stigma in patients, health care workers, care givers and general community in Nicaragua, established that health education intervention directed towards attitude change or knowledge shaping reduced stigma [10]. Other studies on the adherence to treatment for tuberculosis patients have established that patient's demographic characteristics are associated with non-adherence of medication. Maria (2019), on determinants of non-adherence to TB treatment and barriers related to access to treatment in Indonesia, noted an increased risk of non-adherence to treatment in male patients $(\mathrm{OR}=2.8 ; 95 \% \mathrm{Cl} 1.2-6.7)$, patients who had medical check-ups at hospitals $(\mathrm{OR}=3.4 ; 95 \% \mathrm{Cl} 1.1-10.0)$, and those who had difficulties with transportation costs $(\mathrm{OR}=2.5 ; 95 \% \mathrm{Cl} 1.1-5.9)[11]$.

In yet another study on prevalence of, and factors influencing anti-TB treatment non-adherence among patients with pulmonary TB in Anhui Province, Eastern China, Xiu (2019) reported that $33.63 \%$ of 339 patients missed medication; divorced and widowed patients were more likely to miss medication compared to those who were married or unmarried $(p<0.01)$. On the characteristics of the study participants, the mean age was 49 years, and males were $259(76.4 \%)$ while females were $80(23.6 \%)$. In regard to occupation, most of them were farmers (77.0\%). On the education level, $50.15 \%$ were of primary and illiterate levels, $41.89 \%$ were of junior level, $49.49 \%$ of senior high school or technical level, and only $7.96 \%$ were of college level or above [12].

Emesa (2016), in a study whose objective was to measure adherence and determine factors with nonadherence to concurrent TB treatment among co-infected persons in two provinces in South Africa, determined that out of the 1,252 persons receiving concurrent treatment, $138(11.0 \%)$ were not adherent. Non-adherent persons were more likely to have Extra Pulmonary TB (RR: 1.71, 95\% Cl: 1.12 to 2.60) and had not disclosed their Status (RR: 1.96, 95\% Cl: 1.96 to 3.76) [13]. Govender \& Mash (2009) conducted a study in a District Hospital in KwaZulu-Natal, South Africa, to establish the key factors that affected adherence to TB treatment, and recommend interventions that could improve adherence methods. They observed that from the 159 TB Patients, 105 (66\%) were adherent and 54 (34\%) non-adherent. Nonadherence was significantly associated with the level of education, distance from the hospital, time taken to travel, mode of transport and income [14]. 
Habatamu (2018) conducted a study whose aim was to assess the prevalence of non-adherence to antituberculosis treatment, reasons and associated factors among TB patients attending Gondar Town Health Centers in Ethiopia. He noted that the rate of non-adherence to anti-TB therapy was $65(21.2 \%)$ $(95 \% \mathrm{Cl} 17.2,26.1)$. The rate was higher $(47.0 \%)$ among the 'return after default treatment' category and lower (19.1\%) among the 'new' category. In the social-demographic characteristics, with a sample of 314 and $97.5 \%$ response rate, the mean age was 35.94 years, 166 (54.2\%) were male, 135 (44.1\%) were single, 193 (63.0\%) were orthodox Christians, and 75 (24.5\%) were urban dwellers and grade $9-12$ by education. Further, the study established that the income level ( $>3000$ Ethiopian Birr) and patient provider relationship, associated significantly with non-adherence to TB treatment $(0.004,0.004)$ [15].

Fredrick (2004) conducted a study to assess factors contributing to treatment adherence and knowledge of TB transmission among patients on TB treatment in Ndola, Zambia [16]. The study determined that $29.8 \%$ of the TB patients failed to comply with TB drug taking regime once they started feeling better [16]. The study further observed that more females $(39.1 \%)$ than males $(33.9 \%)$ defaulted on medication. The males were older and more educated than the female respondents. However, age, marital status and education levels were not significantly associated with compliance [16].

Muture (2017), on factors associated with default from treatment among TB patients in Nairobi, Kenya, revealed a $16.7 \%$ prevalence of treatment default. Default occurred most frequently during the initial three months of treatment. Among defaulters who were smear positive at initiation of treatment, $47.7 \%$ defaulted before conversion. Factors associated with default included the male sex $(O R 1.43, p<0.001)$ and low income (OR 8.67, $p=0.017)$, among others [17].

\section{Specific Objective}

To determine the effect of Health Education Intervention in promoting adherence to treatment among the urban and rural tuberculosis patients in Kenya.

\subsection{Hypothesis}

Health Education Intervention has no significant effect on adherence to treatment among the urban and rural tuberculosis patients in Kenya.

\subsection{Expected Study Outcome}

To ascertain the difference in Adherence to TB Treatment (Rate/Levels) in the interventional and control groups. This was achieved by collecting baseline data for both the experimental and control groups for reference and end-line data after six months of treatment for both groups. Only the experimental group had an intervention administered to it i.e. the health education program. The changes between the groups were assessed using statistical methods to determine if there was indeed a difference between the two groups after the administration of the intervention.

\section{Methodology}




\subsection{Study design}

The study adopted the Randomized Controlled Trial Design with pre- and post-test data assessments of the TB patients. The TB clinics that were identified for the study were selected randomly. The patients who participated in the study were recruited from the TB attendance and treatment registers in the respective health facilities. Thereafter, they were assigned to experimental and control groups on a 1:1 ratio. The pre-test was conducted on the two groups to establish baseline data of the patients. Health education intervention was then introduced to the experimental group. No intervention was given to the control group. However, both groups continued to receive the normal TB treatment medication. The health education intervention was delivered in the form of individualized health messages and counseling. The health education intervention was conducted during patients' monthly follow-up appointments in outpatients' clinics. The two groups were then followed for the entire period (six months) of TB treatment. After the study period (six months), both groups were then assessed (post-test) to observe the differences in the treatment effect as a result of the health education intervention.

\subsection{Eligibility Criteria of participants}

To qualify as a TB case for inclusion into the study, the patient had to be an adult (18 years and above) undergoing full course of TB treatment and under DOTS Strategy. Further, the patient had to have been registered in the TB attendance and treatment register for at least two weeks in those health facilities. The study excluded patients that were underage (below 18 years), those diagnosed with Multiple Drug Resistant TB, TB patients diagnosed with HIV and those who were already participating in other interventional studies

\subsection{Study setting}

The study was carried out in public health facilities in Nairobi and Murang'a Counties in the Republic of Kenya. Kenya was identified for the study due to its rising TB burden among the high burden countries. Nairobi County was chosen due to its TB burden nationally and its urban setting. Murang'a County was chosen due to its rural setting and a TB prevalence rate similar to that of Nairobi County (4.9\%).

\subsection{Study population}

The study targeted 4149 TB patients who attended TB clinics of the public health facilities in Nairobi and Murang'a counties for treatment. Out of these, Nairobi County had 3319 patients while Murang'a County had 830.

\subsection{Sample size and sampling technique}

The targeted sample size was 450 TB patients, randomly selected from the health facilities which had been identified for the study. The study adopted the Multi-Stage Sampling methodology. Kenya was purposively selected due to its large and rising TB burden. Nairobi County was purposively selected due to its TB burden nationally and its urban setting. Murang'a County was also purposively selected due to its TB prevalence rate (4.9\%) which was similar to that of Nairobi County, and also for its rural setting. Random sampling method was used to select the hospitals, health centers and dispensaries ( 2 hospitals, 
5 health centers and 7 dispensaries $=14$ public health facilities). Random sampling proportionate to TB patients' population was adopted in selecting the study patients (Additional files).

\subsection{Sample Size Determination}

Sample Size was determined by using the Lemeshow \& Hosmer (1990) sample size formula for case controlled studies:

$$
n=\frac{\left(z \frac{\alpha}{2}+Z \beta\right)^{2} * p 1(1-p 1)+p 2(1-p 2)}{(p 1-p 2)^{2}}
$$

- Where $\mathrm{Za} / 2$ is the Critical Value of the Normal Distribution=1.96

- $\mathrm{Z} \beta$ is the Critical Value of the Normal Distribution=1.26

- P1 and p2 are the Expected Sample Proportions of the two Groups.

- $\mathrm{p} 1=85 \%$ (0.85) Proportion of TB Patients who are Adherent according to previously reported result under Normal TB Treatment Strategy (DOTS),

- And by Hypothesizing p2 = 95\% (0.95) and considering 95\% Confidence Interval, 80\% power, 5\% Margin of Error and equal Sample Size for each Group, then the Sample Size will be 137 for each Group.

- To overcome the design effect we assumed Design effect 1.5. The Sample Size was then : $137 \times 1.5$ $=205$

- $10 \%(25)$ of the Sample will be added to take care of Mortality, Transfers and possible Withdrawals.

- Total Sample size (225) for each Group.

- Total Sample size for the two Groups (450)

- Interventional Group (225) and Control Group (225)

From the targeted sample size of 450 TB patients, 370 were targeted from Nairobi and 80 from Murang'a. Further, 225 patients were allotted to the experimental group and a similar number in the control group. For Nairobi County, 185 patients were allotted for the experimental group and a similar number for the control group and in Murang'a County, 40 patients were allotted in the experimental group and 40 for the control group.

\subsection{Patients' recruitment}

TB patients were recruited from the fourteen selected health facilities with the help of the clinical staff in the facilities. In each of these facilities, the TB patient was identified through the TB attendance and treatment registers. To ensure that patients started and ended the health program intervention at the same time, patients who had been on treatment for at least two weeks at the time of selection were recruited. The potential participants were then provided with detailed explanation about the study 
objectives. After assurance of confidentiality those e willing to participate in the study were asked to sign the Informed Consent Form (additional files).I

\subsection{Data collection}

The Standard Questionnaire was the main instrument for data collection from the patients. Data was collected on the social-demographic characteristics of the patients. This comprised age, sex, income, education level, employment, residence, household income and household size. Furthermore, data on self-reported adherence to TB treatment was collecting using the Morisky Medication Adherence Scale (MMAS) (Additional files) which measures the drug taking behavior of the patient and reasons for the patient to likely default on medication (18). To capture the TB Patient case management details, a structured Hospital Data Treatment Form was developed. The key parameters for this review included: Name of Health Facility, TB Case, Date Registered, Age, Sex, Residence, TB Supporter, and Contact Details of the Patient. Based on this information, the patients were divided into experimental and control groups.

\subsection{Ethical Considerations}

Permission was sought from the Kenyatta National Hospital, University of Nairobi Ethics Review Committee, National Commission for Science, Technology and Innovation (NACOSTI), and Nairobi and Murang'a Health Services. The participant's consent was voluntary, free of any coercion, intimidation or inflated promise of benefits from participation. Care was taken to ensure that the consent form was administered by someone who did not hold authority over the participant. Anonymity, Confidentiality, Secrecy and Privacy were safeguarded with regard to information about Treatment, Medical records and drugs for the TB Patient.

\subsection{The Health Education Intervention Program (PRECEDE-PROCEED) Model)}

The PRECEDE-PROCEED Model of study (Additional files) was used to implement the health education intervention activities. The various activities and tasks that were carried out were designed to coincide with the time the Patient was seeking TB treatment. Out of 450 respondents the TB patients were divided into two study groups. The experimental group (225) patients who received the health education intervention and the control group which consisted of (225) patients and who did not receive the health education intervention. TB Patients and their Supporters were engaged in a Health Education Program for 10-15 minutes on average. The Health Education Interaction was twice a month for the next $6+$ months as the Patient went for the weekly drug ration. For better outcomes, the research encouraged team work among the medical/health staff at facility level in the delivery of the health education program particularly in the mobilization of the patients. However, the main researcher took lead in the entire process. The health education technique used was in form of teaching, questions and answers, interview, discussion and scenario analysis. Education materials consisted of interactive tools including pictures and cards with topics for discussion on basic issues about TB. 
The components of the health education provided essential facts about the disease, diagnosis and treatment, potential barriers to treatment adherence, possible adverse effects of the medication, provision of support through counseling and encouragement of social support from family and friends. Pre-test assessment data and post-test assessment data on patient's demographic characteristics (age, sex, education, marital status, occupation, income etc.) and TB medication and adherence were collected at baseline (first month) and end-term (six months) of the study.

\subsection{Statistical methods used}

Both descriptive and inferential statistics were used in analyzing data. SPSS v.20 and excel were used to conduct the analysis. Statistical significance was evaluated at $p<0.05$.

Patient's social/demographic characteristics (age, sex, education level, marital status, occupation, household size and household incomes) were summarized using descriptive statistics. This was presented in tables showing frequencies, standard deviation and percentages.

MANOVA was used to show the effects of the health education program on TB Patient's Medication Adherence. The null hypotheses in each of the objectives were tested using Wilk's $\Lambda$ followed by ANOVA tests for each of the variables. Levene's test for equality of variances was used for assumption testing to determine existing variances in the pre and post-test phases.

Chi-square test and Odds Ratio were also calculated for the post-test results to determine the effectiveness of the health education program and ascertain if the patients subjected to health education were faring better than those who were not subjected to the health education program. Chi-square test was also used to test for the associations between socio-demographic factors and adherence to treatment for the post-test phase results.

\subsection{Assumptions and Limitation of the Study}

The study assumed that all TB cases which were recorded in the counties Public Health facilities and who participated in the study gave the correct information about their social/demographic characteristics, case, treatment and treatment outcomes. It was also assumed that the patients who participated in the study followed the Health Education Intervention Program objectively and honestly shared the effect of the health education experiences. However, there were the possibilities of having obtained less accurate information or biases due to the health state of the patient. Awareness creation and randomization at cluster level ensured bias was minimized.

TB Patients are known to experience stigma. For this reason, discussions about the disease with outsiders may experience some level of rejection. With this knowledge, the researcher worked closely with the TB clinic managers in the health facilities and community health volunteers assigned to the patients.

\subsection{Generalization of the Study}


This was a Randomized Controlled Trial Design study which ensures bias is minimized hence allowing generalization. The review study was conducted in outpatient TB clinics which were similar to the target setting. The participants of the reviewed studies were also comparable to the target audience. The reviewed studies focused on both male and female adults of varied ethnicity. Public health facilities in Kenya attend to patients of similar characteristics. Furthermore, patients in the review studies were similar to the target population in terms of diagnosis and treatment of the disease. The patients in both the review studies and the present study received standard or Prophylactic Treatment for six months. Therefore, the similarity in setting and target populations in both the review studies and our study meant that findings could be generalized the TB Patients in the proposed settings.

\section{Results}

\subsection{Introduction}

The study sought to determine effect of health education in promoting adherence to treatment among the urban and rural TB patients in Kenya. To achieve this goal, the public health facilities that treat TB patients in Nairobi and Murang'a counties were identified for the study. The study took six months with baseline data collected for both groups in September 2019 and end-line data collected from the patients in February 2020 marking the end of the study. The Health Education Interaction was twice a month for the next $6+$ months as the Patient went for the weekly drug ration during the normal course of treatment. All of the patients under review received standard or Prophylactic Treatment for six months thus the study ended after the six months that are standard treatment duration for TB.

\subsection{Response rate}

Though 450 patients were identified and recruited for the study, only 373 patients were included in the study. The remaining 77 did not meet the eligibility criteria due to being underage (less than 18 years), having MDR-TB and those diagnosed with HIV. Of the 373 patients included in the study, 186 patients were in the experimental group and 187 patients were in the control group both at the beginning (baseline) and end (end-line) of the study. None of the patients in the baseline dropped out of the study (Table 1). 
Table 1

Respondents at baseline and end of study

\begin{tabular}{|lll|}
\hline Number of respondents & Baseline & End-line \\
\hline Experimental group & 186 & 186 \\
\hline Control group & 187 & 187 \\
\hline Total & $\mathbf{3 7 3}$ & $\mathbf{3 7 3}$ \\
\hline
\end{tabular}

The targeted respondents for the experimental and control groups were 225 for each. Thus, the response rate for the experimental group was $82.67 \%$ while for the control group, it was $83.11 \%$. The experimental group contributed $49.87 \%$ while the control group represented $50.13 \%$ of the total respondents. The differences in the response rate in both groups were statistically insignificant (Table 2).

Table 2

Response rate

\begin{tabular}{|c|c|c|c|c|c|}
\hline Group & $\begin{array}{l}\text { Number of } \\
\text { respondents }\end{array}$ & $\begin{array}{l}\text { Targeted } \\
\text { respondents }\end{array}$ & Percentage & $\begin{array}{l}\text { Response } \\
\text { rate }\end{array}$ & $\begin{array}{l}\mathrm{p} \\
\text { value }\end{array}$ \\
\hline Experimental & 186 & 225 & $49.87 \%$ & $82.67 \%$ & \multirow[t]{3}{*}{0.859} \\
\hline Control & 187 & 225 & $50.13 \%$ & $83.11 \%$ & \\
\hline Total & 373 & 450 & $100 \%$ & $82.89 \%$ & \\
\hline
\end{tabular}

The targeted respondents for Nairobi County were 370 (185 experimental and 185 control). For Murang'a County they were 80 (40 experimental and 40 control). The actual respondents for Nairobi County were 304 (151 experimental and 153 control) and for Murang'a County, they were 69 (35 experimental and 34 control) (Table 3). 
Table 3

Response rate in the Nairobi and Murang'a facilities

\begin{tabular}{|llll|}
\hline Group & Number of respondents & Targeted respondents & Response rate \\
\hline Nairobi & & & \\
\hline Experimental & 151 & 185 & $\mathbf{8 1 . 6 \%}$ \\
\hline Control & 153 & 185 & $\mathbf{8 2 . 7 \%}$ \\
\hline Murang'a & & & \\
\hline Experimental & 35 & 40 & $\mathbf{8 7 . 5 \%}$ \\
\hline Control & 34 & 40 & $\mathbf{8 5 \%}$ \\
\hline Total & $\mathbf{3 7 3}$ & $\mathbf{4 5 0}$ & $\mathbf{8 2 . 9 \%}$ \\
\hline
\end{tabular}

\subsection{Demographic characteristics of the respondents}

The demographic factors considered included sex, age, level of education, marital status, occupation, and place of residence (urban or rural). As indicated in Table 4 below, $63 \%$ of the respondents were male while $37 \%$ were female. Most (17.4\%) of the respondents were aged between $25-29$ years followed by $15.6 \%$ of respondents aged 30-34 years. Those with primary and secondary school levels of education were $32.5 \%$ and $41.8 \%$. Most of the respondents were household heads $(61.7 \%)$ and were married $(55.5 \%)$. The findings also indicate that most of the respondents were employed in the informal sector $(33.8 \%)$ or unemployed (30\%). Statistical comparison between the experimental and control groups for each of the socio-demographic characteristics showed no differences between the groups, as indicated by the pvalues on the table. 
Table 4

Demographic characteristics of the respondents

\begin{tabular}{|c|c|c|c|c|}
\hline Variable & Experimental & Control & Total & sig(p value) \\
\hline \multicolumn{5}{|l|}{ Sex } \\
\hline Male & $126(67.7 \%)$ & $109(58.3 \%)$ & $235(63 \%)$ & \multirow[t]{3}{*}{0.997} \\
\hline Female & $60(32.3 \%)$ & 78 (41.7\%) & $138(37 \%)$ & \\
\hline Total & 186 & 187 & 373 & \\
\hline \multicolumn{5}{|l|}{ Age Range } \\
\hline Below 20 years & $5(2.7 \%)$ & $9(4.8 \%)$ & $14(3.8 \%)$ & \multirow[t]{9}{*}{0.367} \\
\hline $20-24$ years & $19(10.2 \%)$ & $29(15.5 \%)$ & $48(12.9 \%)$ & \\
\hline $25-29$ years & 37 (19.9\%) & $28(15 \%)$ & $65(17.4 \%)$ & \\
\hline 30-34 years & $31(16.7 \%)$ & $27(14.4 \%)$ & $58(15.6 \%)$ & \\
\hline $35-39$ years & $30(16.1 \%)$ & $21(11.2 \%)$ & $51(13.7 \%)$ & \\
\hline $40-44$ years & 25 (13.4\%) & $28(15 \%)$ & $53(14.2 \%)$ & \\
\hline $45-49$ years & $19(10.2 \%)$ & $15(8 \%)$ & $34(9.1 \%)$ & \\
\hline$\geq 50$ years & $20(10.8 \%)$ & $30(16.1 \%)$ & 50 (13.3\%) & \\
\hline Total & 186 & 187 & 373 & \\
\hline \multicolumn{5}{|l|}{ Level of education } \\
\hline No schooling & $14(7.5 \%)$ & $14(7.5 \%)$ & $28(7.5 \%)$ & \multirow[t]{5}{*}{0.455} \\
\hline Primary school & $61(32.8 \%)$ & $60(32.1 \%)$ & $121(32.5 \%)$ & \\
\hline Secondary school & $78(41.9 \%)$ & $78(41.7 \%)$ & $156(41.8 \%)$ & \\
\hline Tertiary institutions & $33(17.8 \%)$ & $35(18.7 \%)$ & $68(18.2 \%)$ & \\
\hline Total & 186 & 187 & 373 & \\
\hline \multicolumn{5}{|l|}{ Marital Status } \\
\hline Single & $68(36.56 \%)$ & $71(37.97 \%)$ & $139(37.3 \%)$ & \multirow[t]{5}{*}{0.203} \\
\hline Married & 105 (56.45\%) & 102 (54.55\%) & 207 (55.5\%) & \\
\hline Divorced & $7(3.76 \%)$ & $6(3.21 \%)$ & $13(3.5 \%)$ & \\
\hline Separated & $6(3.23 \%)$ & $8(4.27 \%)$ & $14(3.7 \%)$ & \\
\hline Total & 186 & 187 & 373 & \\
\hline Primary occupation & & & & \\
\hline
\end{tabular}




\begin{tabular}{|c|c|c|c|c|}
\hline Variable & Experimental & Control & Total & sig(p value) \\
\hline Agriculture & $20(10.75 \%)$ & 27 (14.44\%) & $47(12.6 \%)$ & \multirow[t]{7}{*}{0.255} \\
\hline Formal sector & $17(9.14 \%)$ & $18(9.63 \%)$ & $35(9.4 \%)$ & \\
\hline Informal sector & $63(33.87 \%)$ & $63(33.69 \%)$ & $126(33.8 \%)$ & \\
\hline Security agencies & $16(8.6 \%)$ & $5(2.67 \%)$ & $21(5.6 \%)$ & \\
\hline students & $13(6.99 \%)$ & 19 (10.16\%) & $32(8.6 \%)$ & \\
\hline Unemployed & 57 (30.65\%) & $55(29.41 \%)$ & $112(30 \%)$ & \\
\hline Total & 186 & 187 & 373 & \\
\hline \multicolumn{5}{|c|}{ Urban and Rural facilities } \\
\hline Nairobi (urban) & $151(81.2 \%)$ & $153(81.8 \%)$ & $304(81.5 \%)$ & \multirow[t]{3}{*}{0.968} \\
\hline Murang'a (rural) & $35(18.8 \%)$ & $34(18.2 \%)$ & $69(18.5 \%)$ & \\
\hline total & 186 & 187 & 373 & \\
\hline \multicolumn{5}{|l|}{ Household head } \\
\hline Yes & $118(63.4 \%)$ & $112(59.9 \%)$ & $230(61.7 \%)$ & \multirow[t]{3}{*}{0.989} \\
\hline No & $68(36.6 \%)$ & $75(40.1 \%)$ & $143(38.3 \%)$ & \\
\hline Total & 186 & 187 & 373 & \\
\hline
\end{tabular}

\subsection{Levels of adherence to TB treatment}

To ascertain levels of adherence, responses in a scale of 8 were scored and grouped into high, low and medium adherence. The Morisky Adherence Medication Scale (MMAS-8) grading was used to assess the non-adherence to medication amongst patients [18]. The MMAS-8 was used by patients to self-report adherence to medication during TB treatment. In compliance with this tool, the levels of adherence were classified as low adherence $(<6)$, medium adherence $(6-8)$ and high adherence $(8)$. According to the $\mathrm{WHO}$, a rate of $80 \%$ is considered high adherence [3].

In the pre-test phase, those with low adherence were $8.6 \%$ (experimental) and $18.7 \%$ (control), while those with high adherence were $32.8 \%$ (experimental) and $17.7 \%$ (control). Majority of the patients in both the experimental (58.6\%) and control (63.6\%) groups had medium adherence. In the post-test phase, majority of the patients had high adherence in both the experimental (83.3\%) and control (60.4\%) groups. Those with low adherence were $1.1 \%$ in the experimental group and $8.6 \%$ in the control group. There was a $50.5 \%$ increase in high adherence patients in the experimental group, and $42.8 \%$ increase in high adherence patients in the control group, indicating that the health education intervention had an impact 
on adherence to TB medication (Fig. 1). In both the low and medium adherence groups, there was a reduction in the proportion observed in the pretest for both the control and treatment groups.

Levels of adherence were further compared between urban (Nairobi) and rural (Murang'a) patients. In urban facilities, in the pre-test phase, majority of the patients were in the medium adherence category in both the experimental (52.98\%) and control groups (65.36\%). In the post-test phase, majority of the patients were in the high adherence category in both the experimental (83.44\%) and control $(67.97 \%)$ groups. There was a $43.05 \%$ increase in high adherence patients in the experimental group while the control group had a $51.63 \%$ increase in high adherence patients (Fig. 2).

In rural facilities, in the pre-test phase, majority of the patients were in the medium adherence category in both the experimental (82.86\%) and control groups (55.88\%). In the post-test phase, however, the majority of patients in the experimental group (82.86\%) were in the high adherence category, while in the control group, majority of the patients (58.82\%) were in the medium adherence category. There was an $82.86 \%$ increase in high adherence patients in the experimental group while the control group had only a $0.94 \%$ increase in high adherence patients. The change in the pre and post-test TB adherence in the experimental group were more enhanced in the rural facilities compared to urban facilities, indicating that the health education intervention had greater impact among rural patients compared to urban patients (Fig. 3).

\subsection{Demographic factors associated with adherence to TB treatment}

All the demographic factors were cross tabulated against the established levels of adherence to TB medication. Table 5 below presents the demographic factors associated with TB medication adherence. The socio-demographic factors of sex and age were not significantly associated with adherence to TB treatment $(p>0.05)$. Level of education $\left(\chi^{2}=27.267, p=0.000\right)$, marital status $\left(\chi^{2}=44.538, p=0.000\right)$, primary occupation $\left(\chi^{2}=18.508, p=0.002\right)$ and place of residence $\left(\chi^{2}=11.782, p=0.001\right)$ were significantly associated with adherence to TB treatment.

In both the experimental and control groups, those with secondary school level of education had the most patients in the high adherence category (88.5\% experimental and $71.8 \%$ control). Similarly, in both groups, those with primary level education and tertiary level education had high adherence levels. Being married was positively associated with high adherence category in both the experimental (93.3\%) and control (65.7\%) groups.

The categories unemployed (91.2\% experimental; $65.5 \%$ control), informal sector (82.5\% experimental; $74.6 \%$ ) and formal sector ( $82.4 \%$ experimental; $50 \%$ control) had the most patients in both the experimental and control groups with slight variations in each group. Urban facilities had more patients $(83.4 \%)$ in the experimental group with high adherence than rural patients $(82.6 \%)$. 
Table 5

Demographic factors associated with TB medication adherence

\begin{tabular}{|c|c|c|c|c|}
\hline Post-test & Variable & Low-medium adherence & High adherence & $\chi^{2}$ sig \\
\hline \multicolumn{5}{|l|}{ Sex } \\
\hline \multirow[t]{2}{*}{ Experimental } & Male & $26(20.6 \%)$ & $100(79.4 \%)$ & \multirow{4}{*}{$\begin{array}{l}\chi^{2}=1.510 \\
p=0.219\end{array}$} \\
\hline & Female & $5(8.3 \%)$ & $55(91.7 \%)$ & \\
\hline \multirow[t]{2}{*}{ Control } & Male & $35(32.1 \%)$ & $74(67.9 \%)$ & \\
\hline & Female & $39(50 \%)$ & $39(50 \%)$ & \\
\hline \multicolumn{5}{|l|}{ Age } \\
\hline \multirow[t]{8}{*}{ Experimental } & Below 20 & $2(40 \%)$ & $3(60 \%)$ & \multirow{16}{*}{$\begin{array}{l}\chi^{2}=13.447 \\
p=0.062\end{array}$} \\
\hline & $20-24$ & $4(21.1 \%)$ & $15(78.9 \%)$ & \\
\hline & $25-29$ & $5(13.5 \%)$ & $32(86.5 \%)$ & \\
\hline & $30-34$ & $4(16.1 \%)$ & $26(83.9 \%)$ & \\
\hline & $35-39$ & $3(10 \%)$ & $27(90 \%)$ & \\
\hline & $40-44$ & $6(24 \%)$ & $19(76 \%)$ & \\
\hline & $45-49$ & $3(15.8 \%)$ & $16(84.2 \%)$ & \\
\hline & $\geq 50$ & $3(15 \%)$ & $17(85 \%)$ & \\
\hline \multirow[t]{8}{*}{ Control } & Below 20 & $6(77.7 \%)$ & $2(22.3 \%)$ & \\
\hline & $20-24$ & $9(31 \%)$ & $20(69 \%)$ & \\
\hline & $25-29$ & $11(39.3 \%)$ & $17(60.7 \%)$ & \\
\hline & $30-34$ & $7(25.9 \%)$ & $20(74.1 \%)$ & \\
\hline & $35-39$ & $9(42.9 \%)$ & $12(57.1 \%)$ & \\
\hline & $40-44$ & $12(42.9 \%)$ & $16(57.1 \%)$ & \\
\hline & $45-49$ & $10(66.7 \%)$ & $5(33.3 \%)$ & \\
\hline & $\geq 50$ & $9(30 \%)$ & $21(70 \%)$ & \\
\hline \multicolumn{5}{|c|}{ Level of education } \\
\hline \multirow[t]{4}{*}{ Experimental } & No schooling & $9(64.3 \%)$ & $5(35.7 \%)$ & \multirow{4}{*}{$\begin{array}{l}\chi^{2}=27.267 \\
p=0.000\end{array}$} \\
\hline & Primary school & $8(13.1 \%)$ & $53(86.9 \%)$ & \\
\hline & Secondary school & $9(11.5 \%)$ & 69 (88.5\%) & \\
\hline & Tertiary & $5(15.2 \%)$ & 28 (84.8\%) & \\
\hline
\end{tabular}




\begin{tabular}{|c|c|c|c|c|}
\hline Post-test & Variable & Low-medium adherence & High adherence & $\chi^{2}$ sig \\
\hline \multirow[t]{4}{*}{ Control } & No schooling & $10(71.4 \%)$ & $4(28.6 \%)$ & \\
\hline & Primary school & $28(46.6 \%)$ & $32(53.4 \%)$ & \\
\hline & Secondary school & $22(28.2 \%)$ & $56(71.8 \%)$ & \\
\hline & Tertiary & $14(40 \%)$ & $21(60 \%)$ & \\
\hline \multicolumn{5}{|l|}{ Marital status } \\
\hline \multirow[t]{4}{*}{ Experimental } & Single & $13(19.1 \%)$ & $55(80.9 \%)$ & \multirow{8}{*}{$\begin{array}{l}x^{2}=44.538 \\
p=0.000\end{array}$} \\
\hline & Married & $7(6.7 \%)$ & $98(93.3 \%)$ & \\
\hline & Divorced & $6(85.7 \%)$ & $1(14.3 \%)$ & \\
\hline & Separated & $5(83.3 \%)$ & $1(16.7 \%)$ & \\
\hline \multirow[t]{4}{*}{ Control } & Single & $28(39.5 \%)$ & $43(60.5 \%)$ & \\
\hline & Married & $35(34.3 \%)$ & $67(65.7 \%)$ & \\
\hline & Divorced & $5(83.3 \%)$ & $1(16.7 \%)$ & \\
\hline & Separated & $6(75 \%)$ & $2(25 \%)$ & \\
\hline \multicolumn{5}{|c|}{ Primary occupation } \\
\hline \multirow[t]{6}{*}{ Experimental } & Agriculture & $4(20 \%)$ & $16(80 \%)$ & \multirow{12}{*}{$\begin{array}{l}\chi^{2}=18.508 \\
p=0.002\end{array}$} \\
\hline & Formal sector & $3(17.6 \%)$ & $14(82.4 \%)$ & \\
\hline & Informal sector & $11(17.5 \%)$ & $52(82.5 \%)$ & \\
\hline & Security agencies & $4(25.1 \%)$ & $12(74.9 \%)$ & \\
\hline & Students & $4(30.8 \%)$ & $9(69.2 \%)$ & \\
\hline & Unemployed & $5(8.8 \%)$ & $52(91.2 \%)$ & \\
\hline \multirow[t]{6}{*}{ Control } & Agriculture & $14(51.8 \%)$ & $13(48.2 \%)$ & \\
\hline & Formal sector & $9(50 \%)$ & $9(50 \%)$ & \\
\hline & Informal sector & $16(25.4 \%)$ & $47(74.6 \%)$ & \\
\hline & Security agencies & $3(60 \%)$ & $2(40 \%)$ & \\
\hline & Students & $13(68.4 \%)$ & $6(31.6 \%)$ & \\
\hline & Unemployed & $19(34.5 \%)$ & $36(65.5 \%)$ & \\
\hline \multicolumn{5}{|c|}{ Place of residence } \\
\hline Experimental & Urban & $25(16.6 \%)$ & $126(83.4 \%)$ & $\chi^{2}=11.782$ \\
\hline
\end{tabular}




\begin{tabular}{|c|c|c|c|}
\hline Post-test & Variable & Low-medium adherence & $\begin{array}{ll}\text { High adherence } & \mathrm{R}^{2}=0,001\end{array}$ \\
\hline & Rural & $6(17.4 \%)$ & $29(82.6 \%)$ \\
\hline \multirow[t]{2}{*}{ Control } & Urban & $49(32 \%)$ & $104(68 \%)$ \\
\hline & Rural & $25(73.4 \%)$ & $9(26.6 \%)$ \\
\hline
\end{tabular}

\subsection{Multivariate Analysis of Variance}

The results were subjected to multivariate analysis of variance to test for the effects of the health education program on adherence to medication. The MMAS-8 variables were the dependent variables in this study while the independent variable was the TB treatment program which had two categories; the experimental (interventional) group who were exposed to the health education intervention and the control group who were not exposed to the health education intervention. Wilk's $\Lambda$ test had an $F$ value of $18.540, p<0.001$ and partial $\eta^{2}$ was 0.522 , indicating that $52.2 \%$ of the changes observed in the experimental group were accounted for by the health education intervention. This clearly indicates overwhelming evidence to reject the null hypothesis that health education has no effect on adherence to TB medication (Table 6).

Table 6

Multivariate tests

\begin{tabular}{|llllll|}
\hline Effect & Value & $\mathbf{F}$ & Hypothesis df & sig & Partial eta squared $\left(\boldsymbol{n}^{2}\right)$ \\
\hline Pillai's trace & 0.522 & 18.540 & 16 & 0.000 & 0.522 \\
\hline Wilk's Lambda $(\Lambda)$ & 0.478 & 18.540 & 16 & 0.000 & 0.522 \\
\hline Hotelling's trace & 1.091 & 18.540 & 16 & 0.000 & 0.522 \\
\hline Roy's largest root & 1.091 & 18.540 & 16 & 0.000 & 0.522 \\
\hline
\end{tabular}

Levene's test of equality of error variances was carried out to test homogeneity of variance assumption. Based on Levene's $F$ tests, the homogeneity of variance was satisfied with all of the variables being statistically significant in the post-test phase further indicating evidence to reject the null hypothesis that health education has no effect on adherence to TB medication (Table 7). Multivariate Analysis of Variance (MANOVA) was carried out modeling various adherence variables to compare the experimental and control groups separately on their pre and post-test scores. In the pre-test phase, the differences between the experimental and control groups were not significant except for the variables "Do you sometimes forget to take your medicine" ( $p=0.002)$, "Have you missed your medication on any days in the past two weeks" ( $p=0.025)$, and "How often do you have difficulty remembering to take all your medication" ( $p=0.000)$. The results for the post-test phase were significant for most variables $(p<0.05)$ 
except for the variable "When you feel like your systems under control, do you sometimes stop taking your medication" which had a p value of 0.054 (Table 7). 
Table 7

Multivariate Analysis of Variance for the MMAS-8 variables

\begin{tabular}{|c|c|c|c|c|c|c|c|}
\hline \multirow[b]{2}{*}{ Variable } & \multicolumn{2}{|c|}{ Levene's test } & \multicolumn{3}{|c|}{ MANOVA } & \multirow{2}{*}{$\begin{array}{l}\text { Experimental } \\
\text { Mean (SD) }\end{array}$} & \multirow{2}{*}{$\begin{array}{l}\text { Control } \\
\text { Mean } \\
\text { (SD) }\end{array}$} \\
\hline & $\mathbf{F}$ & p & $F$ & p & $\begin{array}{l}\text { Partial } \\
\eta^{2}\end{array}$ & & \\
\hline $\begin{array}{l}\text { Do you sometimes } \\
\text { forget to take your } \\
\text { medication (pre-test) }\end{array}$ & 44.024 & 0.000 & 9.786 & 0.002 & 0.033 & $1.94(0.24)$ & $\begin{array}{l}1.82 \\
(0.39)\end{array}$ \\
\hline $\begin{array}{l}\text { Do you sometimes } \\
\text { forget to take your } \\
\text { medication (post- } \\
\text { test) }\end{array}$ & 22.294 & 0.000 & 5.180 & 0.024 & 0.018 & $2.00(0.00)$ & $\begin{array}{l}1.97 \\
(0.18)\end{array}$ \\
\hline $\begin{array}{l}\text { Have you missed } \\
\text { your medication on } \\
\text { any days in the past } \\
\text { two weeks? (pre-test) }\end{array}$ & 21.550 & 0.000 & 5.048 & 0.025 & 0.017 & $1.96(0.2)$ & $\begin{array}{l}1.89 \\
(0.32)\end{array}$ \\
\hline $\begin{array}{l}\text { Have you missed } \\
\text { your medication on } \\
\text { any days in the past } \\
\text { two weeks? (post- } \\
\text { test) }\end{array}$ & 38.249 & 0.000 & 8.470 & 0.004 & 0.029 & $2.00(0.00)$ & $\begin{array}{l}1.94 \\
(0.23)\end{array}$ \\
\hline $\begin{array}{l}\text { Have you ever cut } \\
\text { back on your } \\
\text { medication without } \\
\text { telling the doctor } \\
\text { (pre-test) }\end{array}$ & 14.733 & 0.000 & 3.515 & 0.062 & 0.012 & $1.97(0.16)$ & $\begin{array}{l}1.92 \\
(0.27)\end{array}$ \\
\hline $\begin{array}{l}\text { Have you ever cut } \\
\text { back on your } \\
\text { medication without } \\
\text { telling the doctor } \\
\text { (post-test) }\end{array}$ & 32.685 & 0.000 & 7.357 & 0.007 & 0.025 & $2.00(0.00)$ & $\begin{array}{l}1.95 \\
(0.22)\end{array}$ \\
\hline $\begin{array}{l}\text { When you travel or } \\
\text { leave home, do you } \\
\text { sometimes forget to } \\
\text { bring along your } \\
\text { medication? (pre- } \\
\text { test) }\end{array}$ & 16.002 & 0.000 & 3.821 & 0.052 & 0.013 & $1.93(0.25)$ & $\begin{array}{l}1.86 \\
(0.35)\end{array}$ \\
\hline $\begin{array}{l}\text { When you travel or } \\
\text { leave home, do you } \\
\text { sometimes forget to } \\
\text { bring along your } \\
\text { medication? (post- } \\
\text { test) }\end{array}$ & 68.497 & 0.000 & 14.756 & 0.000 & 0.049 & $1.91(0.29)$ & $\begin{array}{l}1.74 \\
(0.44)\end{array}$ \\
\hline $\begin{array}{l}\text { Did you take all your } \\
\text { medication } \\
\text { yesterday? (pre-test) }\end{array}$ & 2.263 & 0.134 & 0.562 & 0.454 & 0.002 & $1.09(0.29)$ & $\begin{array}{l}1.10 \\
(0.30)\end{array}$ \\
\hline
\end{tabular}




\begin{tabular}{|c|c|c|c|c|c|c|c|}
\hline \multirow[b]{2}{*}{$\begin{array}{l}\text { Did you take all your } \\
\text { medication } \\
\text { yesterday? (post- } \\
\text { test) }\end{array}$} & \multicolumn{2}{|c|}{ Levene's test } & \multicolumn{3}{|c|}{ MANOVA } & \multirow{2}{*}{$\begin{array}{l}\text { Experimental } \\
1.03(0.18)\end{array}$} & \multirow{2}{*}{$\begin{array}{l}\text { Control } \\
1.00 \\
(0.00)\end{array}$} \\
\hline & 21.970 & 0.000 & 5.107 & 0.025 & 0.017 & & \\
\hline $\begin{array}{l}\text { When you feel like } \\
\text { your systems under } \\
\text { control, do you } \\
\text { sometimes stop } \\
\text { taking your } \\
\text { medication? (pre- } \\
\text { test) }\end{array}$ & 12.120 & 0.001 & 2.912 & 0.089 & 0.010 & $1.95(0.22)$ & $\begin{array}{l}1.97 \\
(0.17)\end{array}$ \\
\hline $\begin{array}{l}\text { When you feel like } \\
\text { your systems under } \\
\text { control, do you } \\
\text { sometimes stop } \\
\text { taking your } \\
\text { medication? (post- } \\
\text { test) }\end{array}$ & 15.794 & 0.000 & 3.756 & 0.054 & 0.013 & $1.91(0.29)$ & $\begin{array}{l}1.97 \\
(0.18)\end{array}$ \\
\hline $\begin{array}{l}\text { Do you ever feel } \\
\text { hustled about } \\
\text { sticking to a } \\
\text { medication plan? } \\
\text { (pre-test) }\end{array}$ & 13.694 & 0.00 & 3.445 & 0.064 & 0.002 & $1.76(0.43)$ & $\begin{array}{l}1.66 \\
(0.48)\end{array}$ \\
\hline $\begin{array}{l}\text { Do you ever feel } \\
\text { hustled about } \\
\text { sticking to a } \\
\text { medication plan? } \\
\text { (post-test) }\end{array}$ & 166.633 & 0.000 & 32.404 & 0.000 & 0.101 & $1.91(0.29)$ & $\begin{array}{l}1.65 \\
(0.48)\end{array}$ \\
\hline $\begin{array}{l}\text { How often do you } \\
\text { have difficulty } \\
\text { remembering to take } \\
\text { all your medication? } \\
\text { (pre-test) }\end{array}$ & 43.441 & 0.000 & 15.210 & 0.000 & 0.050 & $1.22(0.55)$ & $\begin{array}{l}1.55 \\
(0.85)\end{array}$ \\
\hline $\begin{array}{l}\text { How often do you } \\
\text { have difficulty } \\
\text { remembering to take } \\
\text { all your medication? } \\
\text { (post-test) }\end{array}$ & 3664.57 & 0.000 & 213.51 & 0.000 & 0.427 & $1.00(0.00)$ & $1.56(0.49)$ \\
\hline
\end{tabular}

\subsection{Analysis of Contingency tables}

Analysis of contingency tables was also carried out to ascertain the association between Health Education on Adherence to treatment. Chi-Squared test indicated strong association between Health Education and Adherence to TB treatment $\left(\chi^{2}=24.189, p<0.001\right)$. Odds ratio calculations also showed that patients who were exposed to Health Education were three times more likely to adhere to treatment 
than those who did not go through the Health Education Intervention (OR 3.274) with 95\% Cl [2.0175.315] (Table 8).

Table 8

Chi-square and Odds ratio

\begin{tabular}{|c|c|c|c|c|c|c|}
\hline \multirow[t]{2}{*}{ Exposure } & & \multicolumn{5}{|c|}{ Adherence } \\
\hline & & High & Medium-low & Total & $\chi^{2}$ sig & OR $(95 \% \mathrm{Cl})$ \\
\hline \multirow[t]{3}{*}{ Experimental } & Count & 155 & 31 & 186 & 24.189 & 3.274 \\
\hline & Expected count & 133.6 & 52.4 & 186 & $(0.000)$ & $(2.017-5.315)$ \\
\hline & \%within exposure & $83.3 \%$ & $16.7 \%$ & $100 \%$ & & \\
\hline \multirow[t]{3}{*}{ Control } & Count & 113 & 74 & 187 & & \\
\hline & Expected count & 134.4 & 52.6 & 187 & & \\
\hline & \%within exposure & $60.4 \%$ & $39.6 \%$ & $100 \%$ & & \\
\hline
\end{tabular}

\section{Discussion}

\subsection{Effect of health education in promoting adherence to tuberculosis treatment in patients}

The instrument used to assess the non-adherence to medication amongst patients was the Morsiky Adherence Medication Scale (MMAS-8) grading. This tool assists the TB patient to self- report adherence to medication during TB treatment. According to this scale, adherence is categorized as low when the scores are $(<6)$, Medium $(6-8)$ and High ( 8$)$. According to WHO (2017) a rate of $80 \%$ and above is considered high adherence [3]. This study established pre-test adherence rates of $32.8 \%$ and $58.6 \%$ for high and medium levels in the experimental group, while in the control group, the adherence levels were $17.7 \%$ and $63.6 \%$ for high and medium levels, respectively. Those with low adherence were $8.6 \%$ in the experimental and control (18.7\%). In the post test phase, patients with high adherence were $83.3 \%$ in the experimental group and $60.4 \%$ in the control. Those with medium adherence were $15.6 \%$ (experimental) and $31 \%$ (control) while those with low adherence were $1.1 \%$ in the experimental and $8.6 \%$ in the control group. These results demonstrate that health education intervention increased adherence in the high adherence category (50\% and $46.7 \%$ ) in both the experimental and control groups. On the other hand, patients in the low category decreased by $7.5 \%$ and $10.1 \%$, respectively. These findings agreed with a study conducted by Pornsak (2016) to investigate the effectiveness of health education program to improve TB patients' compliance during treatment. He established that with intervention, there was $76 \%$ success compared to $62 \%$ in the control group [4]. The findings are also in agreement with the study by Andrew et al. (2019) which established that lack of knowledge regarding TB infection process and its 
treatment contribute to feelings of helplessness and anxiety. With health education intervention, selfreported compliance was high (78\%) against $50 \%$ for the control group [9].

These findings were consistent with the observations of Nagras et al. (2016) in South Africa whose objective was to measure adherence and determine factors associated with non-adherence to concurrent TB treatment and ART, among Co-infected in two provinces. They determined that out of the 1,252 persons receiving concurrent treatment, $138(11.0 \%)$ were not-adherent [5]. Ana et al (2014) in their study in Panoda, Brazil, to establish key factors that affected adherence to TB treatment, recommended interventions that could improve adherence methods observed in 159 patients, 105 (66\%) adhered [6]. Another study by Muture (2017) on factors associated with default from treatment among TB patients in Nairobi Province revealed a $16.7 \%$ prevalence of treatment default. The study further noted that default occurred most frequently during the initial three months of treatment [17]. A study in Iran, whose aim was to assess the prevalence, reasons and associated factors for non-adherence to anti-Tuberculosis treatment among TB Patients attending Gondar town health centers, noted that the rate of non-adherence to anti-TB therapy was $65(21.2 \%) 95 \% \mathrm{Cl} ; 17.2,26.1)$. The rate was higher $(47.0 \%)$ among 'return after default treatment' category and lower (19.1\%) among 'new' category [8].

\subsection{Demographic factors associated with adherence to TB treatment}

Demographic factors that were significantly associated with adherence to TB medication were identified as the level of education, marital status and primary occupation.

\subsubsection{Level of education of the respondents and the adherence to TB treatment}

The study established that adherence to TB treatment improved with high levels of education. Those with secondary school education had better scores (88.5\%) compared to those without schooling (35.7). The high level of education may have contributed to higher levels of health education information uptake among individual patients. These findings agree with a study by Ana et al (2014) that found a significant relationship between levels of education and adherence to TB treatment [6].

\subsubsection{Sex of the respondents and adherence to TB treatment}

In this study the sex of the respondents was not significantly associated with the adherence to TB treatment. However, it was observed that female respondents (91.7\%) adhered more than the males $(67.9 \%)$ after the health education intervention. These findings were in line with those of the study by Edward et al (2014) that assessed factors contributing to treatment adherence and knowledge of TB transmission among patients on TB treatment [10]. They also agree with those of a study by Maria (2019) on determinants of non-adherence to TB treatment and barriers related to access to treatment. Maria's study noted an increased risk of non-adherence to treatment in male patients $(\mathrm{OR}=2.8 ; 95 \% \mathrm{Cl}$ 1.2-6.7) [11].

\subsubsection{Marital status of the respondents and adherence to TB treatment}

The study demonstrated that the married respondents (93.3\%) adhered to medication more than the separated respondents (65.7\%) after the health education intervention. Single respondents (80.9\%) also 
had notably high adherence. This agreed with a study by XUI, H.F. (2019) on prevalence of, and factors influencing anti-TB treatment non-adherence among patients with pulmonary TB. The study observed that divorced and widowed patients were more likely to miss medication compared to those who were married or single $(\mathrm{p}<0.01)$ [12].

\subsubsection{Occupation of the respondents and adherence to TB treatment}

In regard to the primary occupation of the respondents, the TB patients in the formal (82.4\%) and informal (82.5\%) sectors as well as those who are unemployed (91.2\%) showed enhanced adherence to treatment following health education intervention. Those who were students (69.2\%) adhered least. Respondents in the agriculture sector also had notably high adherence (80\%). These findings largely agreed with XUI, H.F. (2019) on prevalence of and factors influencing anti-TB treatment non-adherence among patients with pulmonary TB in China. The findings of this study were that farmers $(77.0 \%)$ adhered more than the other respondents [12].

\subsubsection{Age of the respondents and adherence to TB treatment}

The study determined that there was no significant association between the age of the respondents and the adherence to TB treatment. However, it was observed that the age category 35-39 years (90\%) and age 25-29 with 86.5\% had the highest improvement in adherence to treatment after intervention. This study, however, agreed with the findings by Edward et al. (2014) which assessed factors contributing to treatment adherence and knowledge of TB transmission among patients on TB treatment, and established that age, marital status and education levels were not associated with compliance to TB treatment [10].

\section{Conclusion}

The study concluded that health education intervention enabled the patients to adhere to treatment regime.

\section{Recommendations}

The study recommends the development of a comprehensive and multipronged approach in the provision of health education on TB and the importance of treatment adherence in all health facilities and health care settings providing TB treatment services in Kenya. Additionally, there is need to adopt a more personalized approach involving counseling and support needs targeting marginalized and vulnerable groups such as students, the uneducated, divorced, separated and

widowed patients as well as those in rural areas.

\section{Declarations}

\section{Ethics approval and consent to participate}


Ethical approvals to handle human subjects and to carry out the study in Kenya were sought and obtained from Kenyatta National Hospital/ University of Nairobi Ethics Review Committee and the National Commission for Science, Technology and Innovation (NACOSTI), and Nairobi and Murang'a Health Services. Informed consent to participate in the study were sought and obtained voluntarily from each participant. Care was taken to ensure that the consent form was administered by someone who did not hold authority over the participant.

\section{Consent for publication}

All authors have given their consent to publish this publication

\section{Availability of data and materials}

Data are available from the main author upon reasonable request and after completion and conclusion of the study

\section{Competing interests}

The authors declare that they have no competing interests.

\section{Funding}

This study was fully funded by the main author

\section{Authors' contributions}

HMK initiated the study concept and design, supervised data collection, participated in patient tracing and interviews, cleaned, coded, entered and analysed data and drafted the manuscript. FO, EM, and PW supervised the study and were involved from the concept, design, data acquisition and interpretation of the study findings. FO critically reviewed the methodology, study design and development of data acquisition tools, statistical analysis and provided critical interpretation of results and drafted the manuscript. EWK participated in critical revision of study design and supervision of the study. FO participated in the design of the study, guidance on statistical analysis and results presentation. All authors were involved in drafting of the manuscript, read and approved the final manuscript.

\section{Acknowledgements}

We thank Ronnie Midigo for assisting in technical aspects of the study. We are further grateful to all TB coordinators and health care workers in all the selected TB centres in Nairobi and Muranga counties who made patient respondents' identification and data collection possible. We also appreciate colleagues of the school of the Faculty of Applied Sciences and Technology, Technical University of Kenya

\section{Author Information}


Authors 1, 2 \& 3 are based at the Department of Health Systems Management and Public Health, The Technical University of Kenya, Haile Selassie Avenue, P. O. Box 52428, Nairobi, 00200, Nairobi, Kenya.

Author 4 is based at the Department of Public Health, Kenyatta University, P.O Box 43844, Nairobi, Kenya.

\section{References}

[1] WHO, (2015). Global Strategy and target for TB Prevention, Care and Control, 2015, Geneva, Switzerland.

[2] DCDP\&C, (2017). Division of Communicable Diseases Program and Control-Califonia. Department of Public Health, National TB Prevention Survey, 2017.

[3] WHO, (2017). Multidrug Resistant TB (MDR-TB) update WHO global TB Program, 2017, Geneva.

[4] Pornsak K. (2016). Health Education Program for improving TB Migrants Compliance, Mahidok University, Bangkok, Thailand.

[5] Nargas AA, Leah J, Cecily M, Nguyan NL, Denis F, Emesto J, and Payan N. (2018). Adherence Interventions and Outcomes of TB Treatment. Plos Med 15(7) e1002595; dol: 10.1371/Journal. PMed/1002595

[6] Ana P, Lilian M, Maria N, Elizabeth B, Sandra A. (2014). Health Care Management of TB: Integrating a teaching Hospital into Primary Health Care, Panoda, Brazil.

[7] Yan-Yan Liu (2017). Effects of Comprehensive Nursing Intervention in the Quality of Life and Prognosis of Patients with Smear Positive TB, School of Medicine of Xuchang University, Xuchang, PR China.

[8] Habteyes HT, (2016). Psychological and Behavioral determinants of TB Treatment Non-adherence, and planning Education Interventions to improve Treatment adherence. School of Public Health, Tehran, Iran.

[9] Adrew v. Kathrine F, Roda S, Lori D and Payam N. (2019). The importance of adherence in TB treatment Clinical trials. Plos med 16 (12); e1002884. Dol; 10.1371/Journal, pmed, 1002884

[10] Edward B, Danilo N, Indira M. (2014). Cost-effectiveness of improving health Care to people with HIV in Nicaragua. https/dol.org/10.1155/2014/232046

[11] Maria, B H. (2019). Factors associated with non-adherence during TB Treatment among Patients treated with DOTS Strategy in Jayapura, Indonesia.

[12] Xui, H.F. (2019). Prevalence of and Factors influencing anti-TB Treatment non-adherence among Patients with Pulmonary TB. A Cross-Sectional Study in Anhui Province, Eastern China. 
[13] Emesha, W.M. (2016). Adherence to Concurrent TB treatment and anti-retroviral Treatment among Coinfected persons in South Africa, (2008-2010).

[14] Govender, S. Mash, B. (2009). What are the reasons for Patients not adhering to their Anti-TB Treatment in a South African District Hospital? South Africa.

[15] Habtamu, S.M. (2018). Non-adherence to Anti-TB Treatment, reasons and associated Factors among TB patients attending Gondar Town Health Centres, North West Ethiopia.

[16] Frederick, A.D. (2004). An assessment of factors contributing to Treatment adherence and knowledge of TB Transmission among Patients on TB Treatment, Ndola, Zambia.

[17] Muture, B.N. (2017). Factors associated with Default from Treatment among TB Patients in Nairobi Province. Kenya.

[18] Morisky DE, Ang A, Krousel-Wood M, Ward HJ. Predictive validity of a medication adherence measure in an outpatient setting. J Clin Hypertens 2008; 10: 348-54.

\section{Figures}

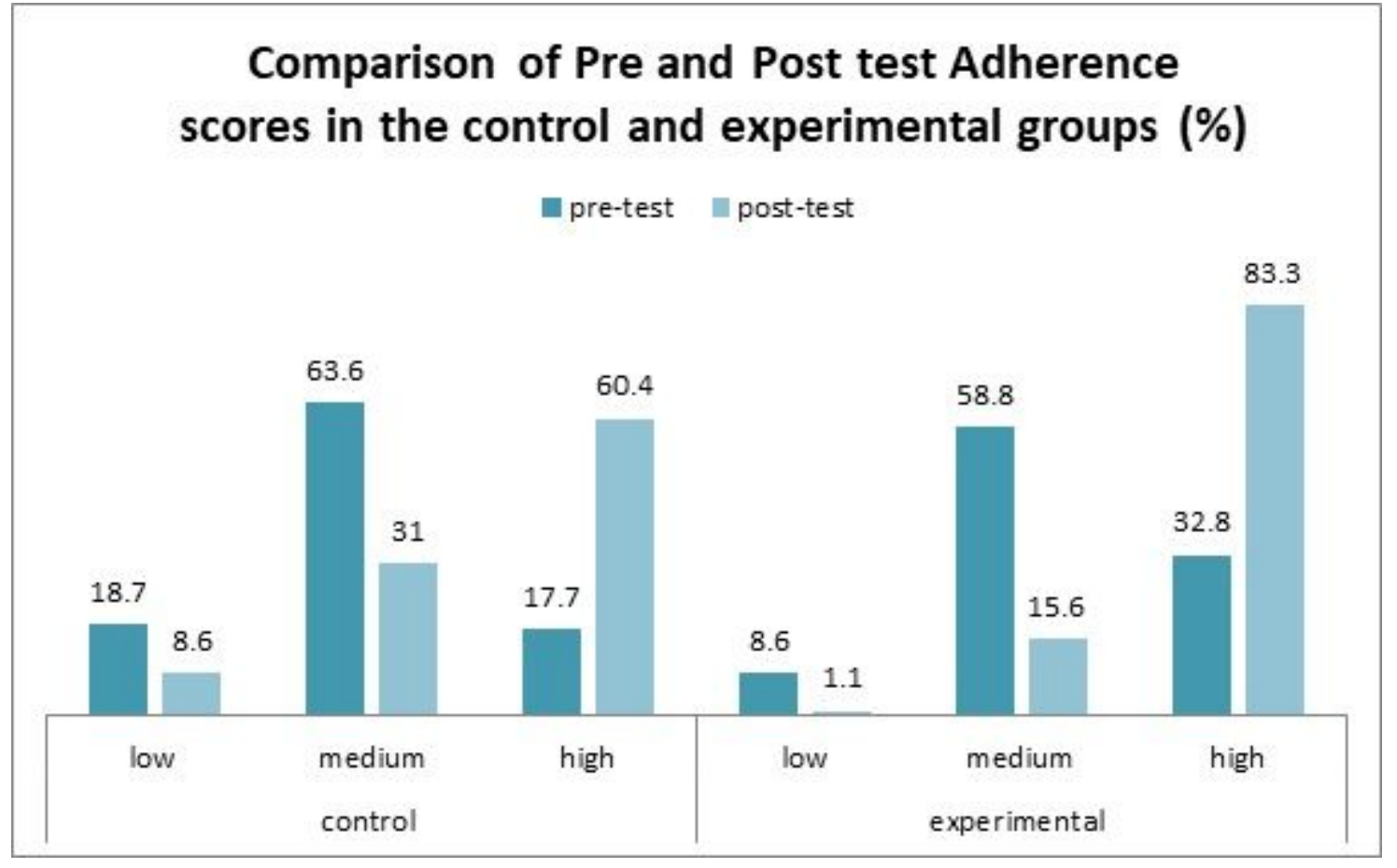

Figure 1

Levels of adherence to TB treatment in the pre-test and post-test phases in the control and experimental groups 


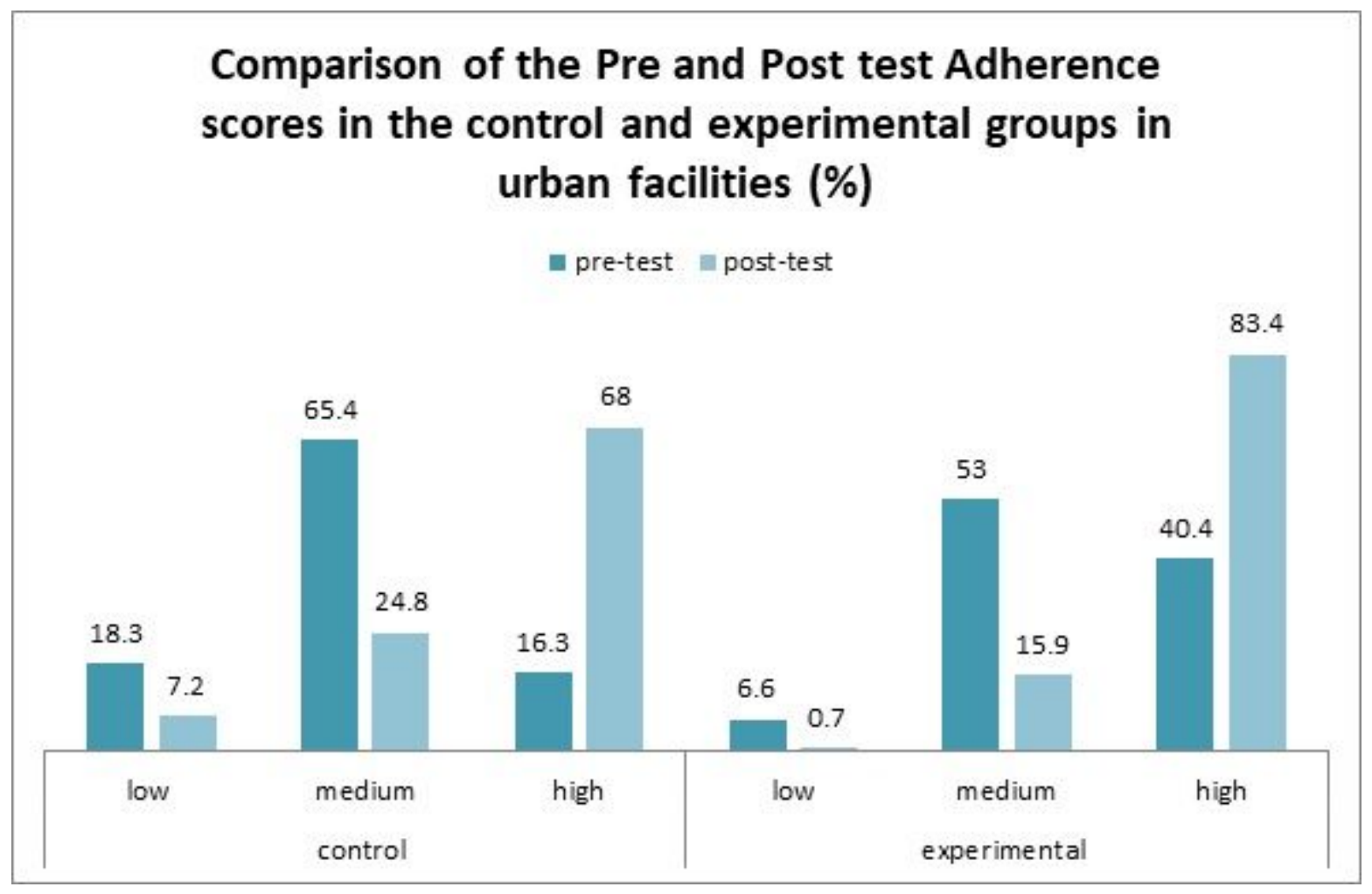

Figure 2

Levels of adherence to TB treatment in the pre-test and post-test phases in the control and experimental groups in Nairobi

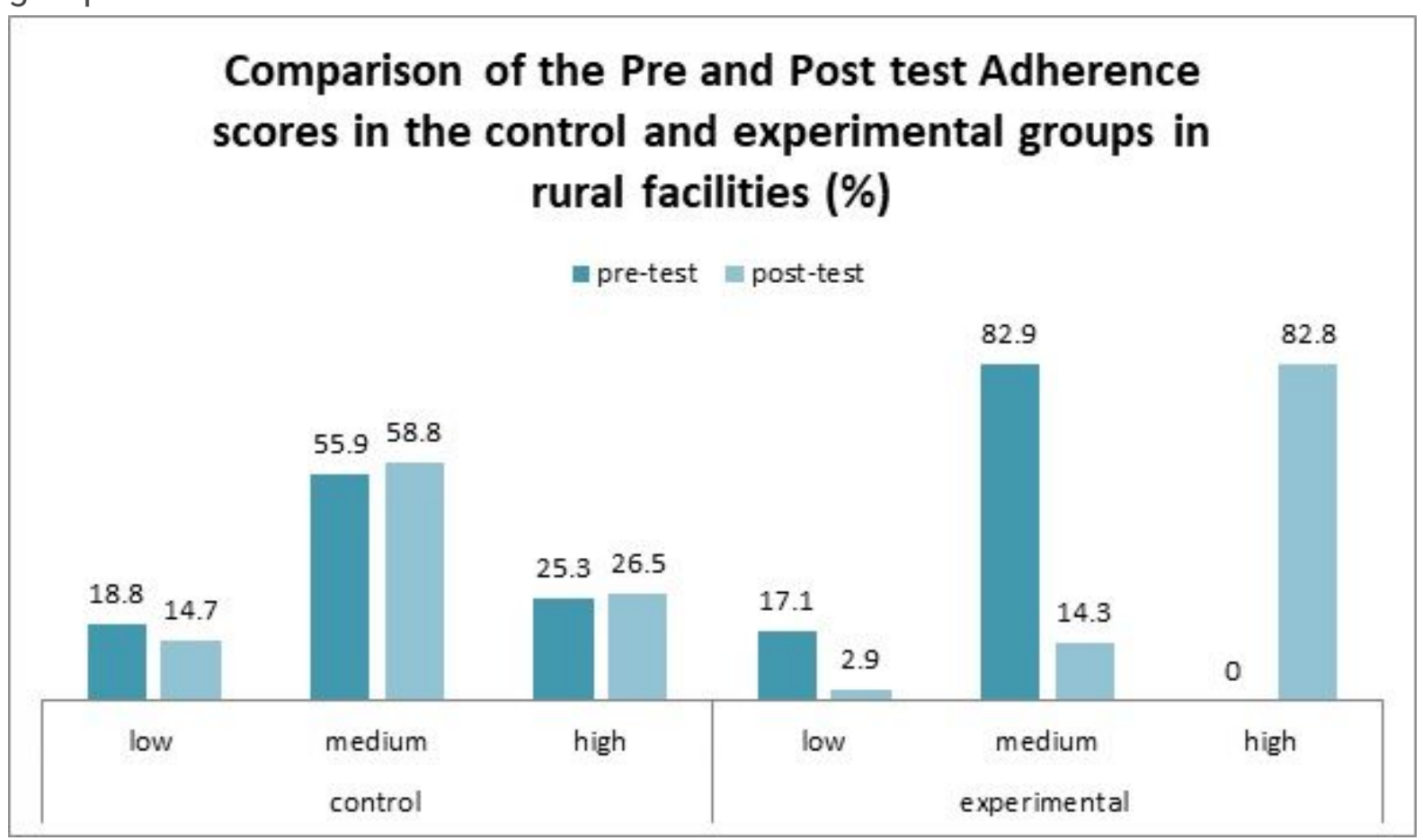

Figure 3

Levels of adherence to TB treatment in the pre-test and post-test phases in the control and experimental groups in Murang'a 


\section{Supplementary Files}

This is a list of supplementary files associated with this preprint. Click to download.

- AdditionalFile.pdf 